\title{
Development of Stable ISFETs for Salivary Nitrate to Acute Stress Monitoring
}

\author{
Shin-ichi Wakida $^{1,2,3}$, Shuto Osaki $i^{1,2}$, Takuya Kintoki $i^{1,5}$, Kenichi Kitamura $^{1,4}$, \\ Koji Murai ${ }^{4}$, Takayo Moriuchi ${ }^{5}$ \\ ${ }^{1}$ AIST PhotoBIO-OIL, National Institute of Advanced Industrial Science and Technology (AIST), \\ 2-1 Yamada-Oka, Suita, Osaka 565-0871, Japan \\ ${ }^{2}$ Graduate School of Human Development and Environment, Kobe University, \\ 3-11 Tsurukabuto, Nada-ku, Kobe 657-8501, Japan \\ ${ }^{3}$ Graduate School of Engineer, Osaka University, 2-1 Yamada-Oka, Suita, Osaka 565-0871, Japan \\ ${ }^{4}$ Graduate School of Maritime Sciences, Kobe University, \\ 5-1-1 Fukae-minami, Higashinada, Kobe 658-0022, Japan \\ ${ }^{5}$ Faculty of Engineering, Osaka Institute of Technology, \\ 5-16-1 Omiya, Asahi-ku, Osaka 535-8585, Japan \\ s.wakida@aist.go.jp
}

\begin{abstract}
:
We studied stable nitrate ion-selective field-effect transistors (ISFETs) to detect human salivary nitrate using direct potentiometry. We investigated several biocompatible polymer based nitrate ISFETs as polymer matrix material. The prepared $\mathrm{NO}_{3}{ }^{-}$-ISFETs showed almost the theoretical Nernst response over $10^{-5.5} \mathrm{M}$ to $10^{-0.5} \mathrm{M}$ with a response time of less than a few seconds except for Tecoflex ${ }^{\circledR}$. As we applied whole human saliva using direct potentiometry, we obtained good relationship with conventional ion chromatography in case of KP-13 based ISFETs. We will also introduce several acute stress subjects for healthy volunteers using wearable heart rate monitor and salivary nitrate by prototype of nitrate FET checkers.
\end{abstract}

Key words: nitrate ISFETs, polyurethane, whole saliva, single drop analysis, direct potentiometry

\section{Introduction}

Stress is defined as the adaptive defense response against changes in the environment as the stress theory. The stress might be evaluated by measuring neurotransmitters in the brain and blood stress hormones. The invasive sampling becomes a very large stress stimulus, and therefore, acute stress cannot be evaluated especially for healthy persons. So, saliva has been attracting attention as an alternative blood sample.

We have carried out several R\&D on single drop analysis for salivary stress marker candidates for ergonomics using microfluidics technology [1]. During the fundamental studies on the prototype of stress-sensing devices, such as electrophoretic microfluidics [2] and ISFETs for real saliva of healthy volunteers, we found salivary nitrate may be a biomarker candidate on the autonomic nervous system response to stressor [3].
We will first introduce stable $\mathrm{NO}_{3}{ }^{-}$-ISFETs for salivary nitrate monitoring using biocompatible polymer matrix materials.

\section{Experimental}

A nitrate-sensing material, nitrate salt of copper (I) bathocuproin (2,9-dibutyl-4,7-di-phenyl-1,10phenanthroline) complex, was synthesized by the conventional solvent extraction method. A plasticizer, 2-nitrophenyldodecyether (NPDDE) was used for stable ISFET to improve the adhesion to gate material of ISFETs [4].

Several biocompatible polymers, calboxylated PVC, PVC-COOH and polyurethane, Tecoflex ${ }^{\circledR}$ were purchased from Aldrich-Sigma and KP-13 [5] was also used for biocompatible polymer as a matrix material.

To evaluate some nitrate-sensitive membranes precisely, nitrate ion-selective electrodes (ISEs) were prepared using an ISE kit (7900-0.65P; TOA-DKK Corp.). Prototype of FET nitrate checkers were prepared by casting the 
tetrahydrofuran solution containing the several nitrate-sensitive polymer membrane materials onto the gate of ISFET device of a pocket-sized $\mathrm{mV}$ Meter (ISFETCOM Co. Ltd.) in the cleanbench as shown in Fig. 1.

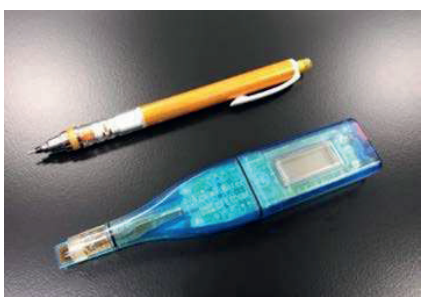

Fig. 1. Photograph of prototype of nitrate checker.

Human saliva was collected using the sampling method with Salivette tubes (SARSTEDT AG \& Co.) or metallic spoon. The saliva sampling experiments were approved by our Institutional Ethics Committee in our institute and informed consent was obtained from all subjects prior to their participation.

\section{Results and discussion}

The prepared $\mathrm{NO}_{3}{ }^{-}$-ISFETs showed stable sensor responses with almost Nernstian slope (ca. $-59 \mathrm{mV}$ per decade change) from $10^{-5.5} \mathrm{M}$ to $10^{-0.5} \mathrm{M}$ with almost Nernst slope were obtained except for Tecoflex ${ }^{\circledR}$.

The stable nitrate-sensing membranes except for Tecoflex ${ }^{\circledR}$, were evaluated using their corresponding ISEs precisely. The $\mathrm{NO}_{3}^{-}$-ISEs showed excellent stable Nernst response over one year with almost the same selectivity obeyed with the Hofmeister series as shown in Fig. 2. The prepared $\mathrm{NO}_{3}{ }^{-}$-ISFETs based checkers showed almost the same sensor characteristics as the corresponding $\mathrm{NO}_{3}{ }^{-}$-ISEs.

The prepared FET nitrate checkers showed fairly stable responses with a rapid response of less than some seconds and so we also applied the human whole saliva using simple metal spoon sampling. We obtained fairly good relationship of the absolute values between the direct potentiometry method and conventional ion chromatography as shown in Fig. 3.
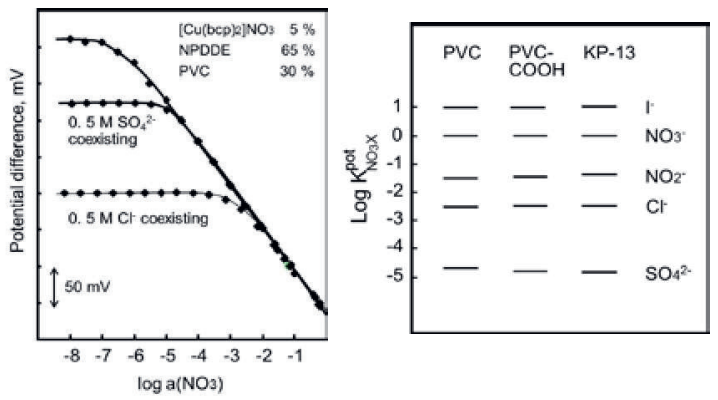

Fig. 2. Static sensor responses of several nitratesensing membranes using $\mathrm{NO}_{3}^{-}-$ISEs.
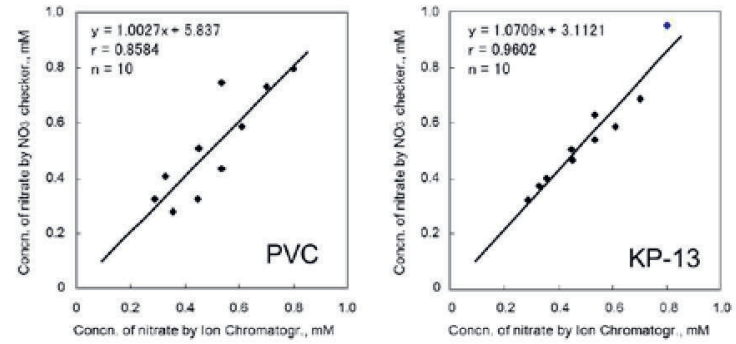

Fig. 3. Correlation between nitrate concentration between nitrate checker and ion chromatography in human whole saliva.

We will also introduce some scientific studies on the acute stress response mechanism of salivary nitrate as the results of simultaneous monitoring for healthy volunteers of several subjects using wearable heart rate monitor and prototype of salivary nitrate checkers [6-8].

\section{References}

[1] S. Wakida, Y. Tanaka, H. Nagai, Research on stress measurement biochip for a single drop of saliva, Folia Pharmacologica Japonica 141, 296 301 (2013); doi: 10.1254/fpj.141.296

[2] T. Miyado, S. Wakida, Rapid stress marker assay using microchip electrophoresis, Rinsho Kensa, 49, 1011-1014 (2005); doi.org/10.11477/mf.1542100251

[3] S. Wakida, Present Status and Perspective View of R\&D on Biochip for Measurement of StressRelated Substances in Saliva, Jpn. J. Stress Sci. 30, 276-284 (2016).

[4] S. Wakida, T. Okumura, Y. Shibutani, J. Liu, Highly Sensitive Nitrate-Sensing Materials for Ion-Selective Field-Effect Transistors for SingleDrop Rain Analysis, Sensor and Materials 19, 235-247 (2007).

[5] S. Wakida, Polymeric Matrix Membrane FieldEffect Transistors, Sodium Ion Sensors for Medical Applications, Biosensors and Chemical Sensors, ACS Symposium Series, 487, Chapter 19, 246-251 (1992); doi: 10.1021/bk-19920487.ch019

[6] K. Kitamura, K. Murai, K. Fukushi, Y. Hayashi, S. Wakida, T. Miyado, N. Mitomo, K. Yoshimura, K. Hikida, Evaluation of Ship Navigator's Mental Workload for Ship Handling Based on Salivary Nitrate. Proc. 2012 IEEE Int. Conf. on Systems, Man, Cybernetics, 1531-1535 (2012); doi: 10.1109/ICSMC.2012.6377953

[7] K. Kitamura, K. Murai, S. Wakida, Evaluation of Mental Workload for a Newly- Appointed Pilot Using Salivary $\mathrm{NO}_{3}{ }^{-}$Concentration and LF/HF Values, Compared with Port-Coordinators, Proc. IFSA-SCIS 2017, 17139698 (2017); doi: 10.1109/IFSA-SCIS.2017.8023280

[8] K. Kitamura, K. Murai, S. Wakida, M. A. Rooks, A Ship Evaluation of Mental Workload for PortCoordinator on an Individual Scale Using Salivary $\mathrm{NO}_{3}{ }^{-}$, Transactions of Navigation 2, 15-23 (2017); doi: org/10.18949/jintransnavi.2.1_15 